\title{
A MINIMAL SWITCHING PROCEDURE FOR CONSTRAINED RANKING AND SELECTION
}

\author{
Christopher M. Healey \\ APC by Schneider Electric \\ 85 Rangeway Rd, Bldg 2 \\ North Billerica, MA 01824, USA
}

\author{
Sigrún Andradóttir \\ Seong-Hee Kim \\ H. Milton Stewart School of Industrial and Systems Engineering \\ Georgia Institute of Technology \\ Atlanta, GA 30332, USA
}

\begin{abstract}
Constrained ranking and selection aims to select the best system according to a primary performance measure, while also satisfying constraints on secondary performance measures. We introduce a new procedure that makes a valid selection of the best constrained system, while minimizing the number of switches between systems. Analytical and experimental results show that the procedure is both valid and efficient in terms of total cost (incorporating both switching and sampling costs).
\end{abstract}

\section{INTRODUCTION}

Ranking and selection (R\&S) procedures are statistical tools for selecting the best system out of a finite number of simulated alternatives. Many approaches exist to address this general problem, either through the indifference-zone method (e.g., Rinott 1978, Kim and Nelson 2006), the Bayesian method (e.g., Chick and Inoue 2001, Chick 2006), or the optimal computing budget allocation (OCBA) method (e.g., Chen et al. 2000).

We consider a more complicated form of $R \& S$, namely selecting the best system that satisfies constraints on one or more secondary performance measures. This problem is known as constrained R\&S. To accomplish this, we adopt the framework of Andradóttir and Kim (2010) that involves a feasibility check phase (to ensure that the chosen system meets the required constraints) and a comparison phase (to determine the best feasible system). Within this framework, we seek to ensure a desired probability of correct selection (PCS) of the best feasible system.

The problem of constrained R\&S has attracted some attention lately, including the development of the fullysequential indifference-zone procedures of Andradóttir and Kim (2010) and Healey et al. (2010a,b), the OCBA methods of Pujowidianto et al. (2009), the multiple attribute theory framework of Morrice and Butler (2006), and an indifferencezone approach that considers constraint feasibility by Kabirian and Ólafsson (2009). In addition, some research has been dedicated to feasibility check alone, as in Batur and Kim (2010) and Szechtman and Yücesan (2008).

The previously mentioned procedures for constrained R\&S aim for efficiency in terms of observations required to find the best feasible system, but there are none that we know of that address the cost of switching between systems explicitly. While it is common to compare procedures based on the required number of samples to achieve a nominal PCS, the possibly high cost (in both time and storage) of stopping and restarting complex simulations should also be considered. Hong and Nelson (2005) and Osogami (2009) present fully-sequential procedures that perform valid comparison while limiting the number of switches. We build on their work and present a new fully-sequential indifference-zone procedure, named the Constrained Minimal Switching (CMS) procedure, that addresses the concern of switching costs, while identifying the best feasible system.

This paper is organized as follows. Section 2 outlines the problem of constrained R\&S, details notation, and sets assumptions for the validity of our procedure. Section 3 introduces the CMS procedure and addresses its validity. Section 4 features experimental results, followed by conclusions in Section 5.

\section{BACKGROUND}

The goal of constrained $R \& S$ is the selection of the best system according to a primary performance measure out of a fixed number of alternatives, $k$, with constraints on $s$ secondary performance measures. We outline the problem in Section 2.1, and introduce notation necessary for our algorithm and its proof in Section 2.2. 


\subsection{Problem Formulation}

Let $\left(X_{i n}, Y_{i 1 n}, \ldots, Y_{i s n}\right)$ be the $n$th observation of the $i$ th system for the primary performance measure and $s$ secondary performance measures. We consider the set of all possible systems $S=\{1, \ldots, k\}$. We let $x_{i}=\mathrm{E}\left[X_{i n}\right]$ and $y_{i \ell}=\mathrm{E}\left[Y_{i \ell n}\right]$ be the mean values of the primary and secondary performance measures for each system $i \in S$ and constraint $\ell=1, \ldots, s$. Therefore our objective is to determine which system has the best primary performance measure, while also satisfying all constraints:

$$
\begin{aligned}
& \arg \max _{i \in S} x_{i} \\
& \text { s.t. } y_{i \ell} \leq q_{\ell} \text { for all } \ell=1, \ldots, s .
\end{aligned}
$$

We let $\quad{ }_{x_{i}}^{2}=\operatorname{Var}\left[X_{i n}\right]$ for all $i$ and $\quad{ }_{y_{i \ell}}^{2}=\operatorname{Var}\left[Y_{i \ell n}\right]$ for all $i$ and $\ell$. The relationship between performance measures is governed by the following assumption. The normality of data is a common assumption within ranking and selection, achieved through within-replication averages or batched means (Law and Kelton 2000).

Assumption 1. For each $i=1,2, \ldots, k$,

$$
\left[\begin{array}{c}
X_{i n} \\
Y_{i 1 n} \\
\vdots \\
Y_{i s n}
\end{array}\right] \stackrel{i i d}{\sim} M N\left(\left[\begin{array}{c}
x_{i} \\
y_{i 1} \\
\vdots \\
y_{i s}
\end{array}\right], i\right) n=1,2, \ldots
$$

where $\stackrel{i i d}{\sim}$ denotes independent and identically distributed, MN denotes multivariate normal, and ${ }_{i}$ is the $(s+1) \times(s+1)$ covariance matrix of the vector $\left(X_{i n}, Y_{i 1 n}, \ldots, Y_{i s n}\right)$.

The procedure detailed in this paper utilizes the indifference-zone method for both the feasibility check and comparison phases. For all systems involved in the simulation, we designate the indifference-zone parameter, , as the smallest significant difference between systems' primary performance measures. So, we are "indifferent" between systems that have means within of each other.

Likewise, we consider the tolerance level $\ell$ to be the smallest significant difference between $y_{i \ell}$ and $q_{\ell}$. Therefore, we can place all systems into three sets in terms of feasibility. If system $i$ is in $S_{D}$, the set of desirable systems, then $y_{i \ell} \leq q_{\ell}-{ }_{\ell}$ for all $\ell=1, \ldots, s . S_{U}$ is the set of undesirable systems where at least one secondary performance measure, $y_{i \ell}$, is infeasible, so that $y_{i \ell}>q_{\ell}+\ell$. All systems not in $S_{D}$ or $S_{U}$ fall into $S_{A}$, the set of acceptable systems.

Assumption 2. Let $x_{[b]} \geq x_{i}+$ for all $i \in S_{D} \cup S_{A} \backslash\{[b]\}$, where $[b]$ is the index of the best feasible system.

Under Assumption 2, we let CS be the correct selection event that system $[b]$ is declared feasible and all systems in $S \backslash\{[b]\}$ are eliminated. If all systems are infeasible, then CS is the event that all systems in $S$ are eliminated. We desire to ensure a nominal PCS at least $1-$.

\subsection{Notation and Assumptions}

We present the following notation:

$n_{0}=$ the first-stage sample size;

$S_{X_{i j}}^{2}=$ the sample variance of the paired difference of $\left\{X_{i 1}, \ldots, X_{i n_{0}}\right\}$ and $\left\{X_{j 1}, \ldots, X_{j n_{0}}\right\}$;

$S_{Y_{i \ell}}^{2}=$ the sample variance of $\left\{Y_{i \ell 1}, \ldots, Y_{i \ell n_{0}}\right\}$ (the $\ell$ th constraint of system $i$ );

$Y_{\text {in }}=\left(Y_{i 1 n}, Y_{i 2 n}, \ldots, Y_{i s n}\right)^{T}$;

$R(r ; a, b, d)=\max \left\{0, \frac{b d}{2 a}-\frac{a}{2} r\right\}$, for $a, b, d \in \mathbb{R}^{+}$and $a \neq 0$;

$C S_{i}=$ the event that a good selection is made in pairwise comparison of systems $i$ and $[b]$, for any $i \in S_{D} \cup S_{A}$ with $x_{[b]} \geq x_{i}+;$

$C D_{i}=$ the event that correct decision is made on the feasibility of system $i \in S$ (when $i \in S_{A}, C D_{i}$ can be infeasible or feasible);

$1=$ the error of an individual feasibility check for one performance measure of one system;

$2=$ the error of an individual comparison between two systems.

With this notation, we now present two assumptions that govern good feasibility check and comparison phases. Assumptions 3 and 4 ensure that feasibility check and comparison are handled in a valid manner.

Assumption 3. The systems are simulated independently, and the feasibility check phase guarantees $\operatorname{Pr}\left\{\cap_{i \in S^{\prime}} C D_{i}\right\} \geq$

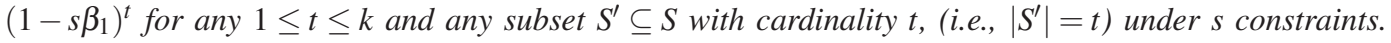


Assumption 4. The systems are simulated independently, and the comparison phase guarantees $\operatorname{Pr}\left\{\cap_{i \in S^{\prime}} C S_{i}\right\} \geq(1-2)^{t}$ for any $1 \leq t \leq k-1$ and any subset $S^{\prime}$ of $\left\{i \in\{1, \ldots, k\}: x_{i} \leq x_{[b]}-\right\}$ with cardinality $t$ (i.e., $\left|S^{\prime}\right|=t$ ).

\section{THE CONSTRAINED MINIMAL SWITCHING PROCEDURE}

In this section, we present a new approach for constrained $\mathrm{R} \& \mathrm{~S}$, namely $C M S$, that minimizes the cost of switching from one system to another. This cost is often not factored into R\&S studies, but it can comprise a large portion of the computation time, as discussed in Hong and Nelson (2005) and Osogami (2009). The feasibility check phase of $C M S$ is performed by the $F_{B}^{I}$ procedure of Batur and Kim (2010) (with $c=1$ ), a general, fully-sequential, and valid method for determining feasibility of multiple constrained performance measures. The comparison phase of CMS is performed by the MSS procedure of Hong and Nelson (2005), modified as described in their Remark 3. The procedure will visit each system at most once after the first stage. To achieve this, at least one system must receive a large number of samples, the maximum necessary to complete comparison with all other systems. Therefore, we expect this algorithm to be conservative in terms of observations, but a good choice if switching costs are high.

The $C M S$ procedure consists of three steps, namely sorting the systems by primary performance measure after the first-stage of sampling, performing feasibility check on systems according to their sorted order to find the initial guess for the best feasible system $(B)$, and then comparing the current guess for the best feasible system $(B)$ with the next best available system $(A)$, until no systems remain. Sampling occurs for only the next best available system $A$. Each successive system $A$ is simultaneously tested for feasibility and compared to $B$. System $A$ can become the current guess for best feasible system only if it is found feasible and superior to system $B$. If one of these conditions is found not to be true, $A$ is eliminated, a new $A$ is chosen to be the next available system, and sampling shifts to the new system $A$. This proceeds until all available systems are eliminated by comparison or feasibility check.

\section{Procedure [CMS for Multiple Constraints]}

Setup: Select the overall confidence level $1 / k \leq 1-\quad<1$ and first-stage sample size, $n_{0} \geq 2$. Choose , $\ell$, and $q_{\ell}$ for $\ell=1,2, \ldots, s$. Let $1=\frac{1}{2}\left(\left(2_{1}\right)^{-2 /\left(n_{0}-1\right)}-1\right)$ and $2=\frac{1}{2}\left(\left(\begin{array}{ll}2 & 2\end{array}\right)^{-2 /\left(n_{0}-1\right)}-1\right)$, where $1_{1}=2 / s$ and 2 is the unique solution to the equation $2+2\left[1-(1-2)^{(k-1) / 2}\right]=$.

Initialization: Let $h_{1}^{2}=2_{1}\left(n_{0}-1\right)$ and $h_{2}^{2}=22_{2}\left(n_{0}-1\right)$. Obtain $n_{0}$ observations $X_{i n}$ and $Y_{\text {in }}$ from each system $i \in S$. For all $i$ and $\ell$, compute the estimators $S_{Y_{i \ell}}^{2}$. Similarly, for all $i$ and $j \neq i$, compute the estimator $S_{X_{i j}}^{2}$. Also compute $N_{i j}$ for all $i, j \in S$ and $i \neq j$, where

$$
N_{i j}=\max \left\{n_{0},\left\lceil\frac{h_{2}^{2} S_{X_{i j}}^{2}}{2}\right\rceil\right\}
$$

and $\lceil\cdot\rceil$ is the ceiling function. Let $S I_{i}=\emptyset$ be the set of systems inferior to system $i$ in terms of the primary performance measure. Let $K_{i}=\emptyset$ be the set of constraints found to be feasible for system $i \in S$ and let the set of contending systems include all systems, $M=S$. The procedure will require the calculation of the maximum samples required for system $i$ to complete comparison with all systems remaining in contention:

$$
N_{i}=\max _{j \in M \backslash\left(S I_{i} \cup\{i\}\right)} N_{i j}
$$

Set the observation counters $r_{i}=n_{0}$ for all $i$.

Finding a Feasible System:

Initial Sorting: Sort the systems in $M$ based on the first-stage sample means $\bar{X}_{i}=\frac{1}{n_{0}}{ }_{n=1}^{n_{0}} X_{i n}$. Let $B$ and $A$ be the systems in $M$ with the best and second-best first stage sample means.

Initial Screening for Comparison: Compare all systems $i \neq j$ in $M$ based on $n_{0}$ samples. If

$$
{ }_{n=1}^{n_{0}} X_{i n} \geq{ }_{n=1}^{n_{0}} X_{j n}+R\left(n_{0} ;, h_{2}^{2}, S_{X_{B j}}^{2}\right),
$$

then add $j$ to $S I_{i}$. Compute $N_{B}$ using (1).

Initial Feasibility Check: For system $B$ and $\ell \notin K_{B}$, if

$$
{ }_{n=1}^{r_{B}}\left(Y_{B \ell n}-q_{\ell}\right) \geq R\left(r_{B} ; \ell, h_{1}^{2}, S_{Y_{B \ell}}^{2}\right),
$$


declare $B$ to be infeasible. Else if

$$
{ }_{n=1}^{r_{B}}\left(Y_{B \ell n}-q_{\ell}\right) \leq-R\left(r_{B} ; \ell, h_{1}^{2}, S_{Y_{B \ell}}^{2}\right)
$$

add $\ell$ to $K_{B}$. If $\left|K_{B}\right|=s$, declare $B$ to be feasible, remove all systems in $S I_{B}$ from $M$, and update $A$, if necessary. Stopping Rule: If $B$ is feasible and $|M|=1$, declare $B$ as the best feasible system. If $B$ is infeasible and $|M|=1$, then no feasible systems exist. If $B$ is feasible and $|M|>1$, proceed to Feasibility and Comparison of $A$ with $B$. If $B$ is infeasible and $|M|>1$, then remove $B$ from $M$, set $B=A$, compute $N_{B}$ using (1), let $A$ be the best system in $M \backslash\{B\}$ if $M \backslash\{B\} \neq \emptyset$, and proceed to Initial Feasibility Check. Otherwise, take an additional sample from system $B, X_{B, r_{B}+1}$ and $Y_{B, r_{B}+1}$, and set $r_{B}=r_{B}+1$. If $r_{B}=N_{B}$, store ${ }_{n=n_{0}+1}^{N_{B}} X_{B n}$. Go to Initial Feasibility Check.

\section{Feasibility and Comparison of $A$ with $B$ :}

Sampling for Comparison: Find $N_{A}$ using (1). If $r_{B}<N_{B}$, take an additional $N_{B}-r_{B}$ observations from system $B$ and set $r_{B}=N_{B}$.

Comparison: If $B \notin S I_{A}$ and

$$
\frac{r_{A}-n_{0}}{N_{B}-n_{0}}{ }_{n=n_{0}+1}^{N_{B}} X_{B n}+{ }_{n=1}^{n_{0}} X_{B n} \geq{ }_{n=1}^{r_{A}} X_{A n}+R\left(r_{A} ;, h_{2}^{2}, S_{X_{B A}}^{2}\right),
$$

then remove $A$ from $M$ and go to Stopping Rule.

If $B \notin S I_{A}$,

$$
\frac{r_{A}-n_{0}}{N_{B}-n_{0}}{ }_{n=n_{0}+1}^{N_{B}} X_{B n}+{ }_{n=1}^{n_{0}} X_{B n} \leq{ }_{n=1}^{r_{A}} X_{A n}-R\left(r_{A} ;, h_{2}^{2}, S_{X_{B A}}^{2}\right),
$$

and $A$ is feasible, then remove $B$ from $M$. If $B \notin S I_{A},(2)$ is true, and $A$ 's feasibility is undetermined, add $B$ to $S I_{A}$. Feasibility: If the feasibility of $A$ is unknown, use the same procedure as Initial Feasibility Check, except substitute $A$ for $B$. If $A$ is feasible, remove all system in $S I_{A}$ from $M$. If $A$ is infeasible, eliminate system $A$ from $M$. Stopping Rule: If $|M|=1$, stop and declare the remaining system as the best. If $B \notin M$, then set $B=A$, update $A$, and go to Sampling for Comparison. If $A \notin M$, update $A$ and go to Sampling for Comparison. Otherwise, take an additional sample from system $A, X_{A, r_{A}+1}$ and $Y_{A, r_{A}+1}$, and set $r_{A}=r_{A}+1$. If $r_{A}=N_{A}$, store ${ }_{n=n_{0}+1}^{N_{A}} X_{A n}$. Go to Comparison.

Note that the $C M S$ procedure utilizes only $N_{B}$ samples for comparison, even if more samples are obtained in a long feasibility check. This is desirable because Healey et al. (2010a) show that primary performance measure sample means may be biased at the completion of feasibility check if primary and secondary performance measures are correlated, so observations past $N_{B}$ are possibly harmful.

We now present the main result in this section. Healey et al. (2010c) provide the complete proof. Note that for fixed $k, 2 \times\left[(1-2)^{(k-1) / 2}\right]-2-1$ monotonically decreases from 1 to -2 as 2 increases from 0 to 1 , guaranteeing a unique solution to equation (3) below.

Theorem 1. When the systems are simulated independently and Assumptions 1 and 2 hold, CMS guarantees

$$
\operatorname{Pr}\{C S\} \geq 1-
$$

when

$$
2 \times\left[(1-2)^{(k-1) / 2}\right]-2-1=1-
$$

\section{EXPERIMENTAL RESULTS}

In this section, we evaluate the performance of our new $C M S$ procedure compared to the performance of another constrained R\&S procedure, HAK+ of Healey et al. (2010b), in terms of the number of switches, number of required observations, and observed PCS. $H A K+$ is a simultaneously-running procedure that performs both feasibility check and comparison on all systems remaining in contention after each stage of sampling. This procedure extends the $A K+$ procedure of Andradóttir and Kim (2010) to incorporate multiple constraints. In Section 4.1, we discuss the experimental setup for all of our tests. We provide an analysis of $C M S$ in a small set of experiments in Section 4.2. 


\subsection{Setup}

Our experiments will test the procedures in two different combinations of means with 10,000 macro-replications. The configurations will consider three constrained performance measures. We specify $\quad \underset{x_{i}}{2}=1$ and $\underset{y_{i \ell}}{2}=1$. We set $n_{0}=20$, and and $\ell$ equal to the sample standard deviation $1 / \sqrt{20}$ of the average when samples have a variance of 1 for all $\ell=1,2,3$. The nominal PCS is $1-\quad=0.95$. We set the number of acceptable system in $S_{A}$ to be zero, as Andradóttir and Kim (2010) show that the existence of acceptable systems does not affect results significantly.

The difficult means configuration (DM) attempts to test the validity of the procedures by assigning system means in the most challenging setup. Systems are placed into two groups with respect to the best feasible system $b$ : some systems are only slightly inferior, but also feasible by a small amount, and some systems are vastly superior and also only slightly infeasible. All infeasible systems have one constraint that is violated. Hence, in the DM configuration,

$$
x_{i}=E\left[X_{i n}\right]= \begin{cases}0, & i=1,2, \ldots, b-1, \\ , & i=b \\ (i-1), & i=b+1, \ldots, k\end{cases}
$$

and

$$
y_{i \ell}=E\left[Y_{i \ell n}\right]=\left\{\begin{array}{cl}
-\ell, & i=1,2, \ldots, b \\
\ell, & i=b+1, \ldots, k \text { and } \ell=1 \\
-\ell & i=b+1, \ldots, k \text { and } \ell=2,3
\end{array}\right.
$$

We set the constraint levels, $q_{\ell}$, to zero.

We also consider the MIM configuration that will allow us to determine the efficiency at which the procedures determine the feasibility of clearly infeasible or feasible systems and compare substantially distant systems. In the MIM configuration,

$$
x_{i}=E\left[X_{i j}\right]=(i-1), \quad i=1,2, \ldots, k,
$$

and

$$
y_{i \ell}=E\left[Y_{i \ell j}\right]= \begin{cases}-(b-i+1) \ell, & i=1,2, \ldots, b, \\ (i-b) \ell, & i=b+1, \ldots, k, \text { and } \ell=1, \\ -(i-b) \ell, & i=b+1, \ldots, k, \text { and } \ell=2,3,\end{cases}
$$

where again we set $q_{\ell}=0$.

To illustrate the combined cost of sampling and switching for our systems, we present the total cost as the combined cost of observations and switches. Hong and Nelson (2005) perform an analysis of total costs when switching costs are a factor of 1, 10, 100, or 1000 times larger than the sampling costs per observation. We feature experimental results for the first factor, 1; the other three factors will yield results that are more favorable to CMS.

Systems are simulated independently. Andradóttir and Kim (2010) and Healey et al. (2010a) present empirical results that show that the correlation across primary and secondary performance measures does not have a major impact on performance, so we will not revisit that topic in this paper. Similarly, Batur and Kim (2010) show that correlation across secondary performance measures does not largely affect the performance of the feasibility check procedure $F_{B}^{I}$. We expect similar conclusions would be found here, and hence implement our procedures with independent secondary performance measure samples.

\subsection{Results}

In our experimental results, we display the effectiveness of multiple constrained R\&S procedures, with respect to observed PCS, average number of required samples, and average number of switches, while defining a switch to be the initialization and resuming of sampling for a system. We operate the two procedures under similar setups. For example, we choose $1 / s=2$ in $H A K+$ and $C M S$, so that error is allocated equally between feasibility check and comparison.

Tables 1 and 2 display the observed PCS, average number of observations, and average number of switches, respectively, for 15 systems with 8 feasible for the DM and MIM configurations, respectively. We choose $b=\left\lceil\frac{k+1}{2}\right\rceil$ to minimize the PCS of our procedures. This setup challenges the PCS of the procedures, as shown by Andradóttir and Kim (2010) and Healey et al. (2010b).

The comparison phase of $C M S$ makes this procedure less attractive than $H A K+$ with respect to the number of required observations, as seen in Tables 1 and 2. In Table 1, CMS requires about $45 \%$ more observations than $H A K+$. Similarly, under the MIM configuration in Table 2, CMS again requires $45 \%$ additional observations. 
Healey, Andradóttir and Kim

Table 1: Average number of required observations (OBS), observed PCS, average number of required switches (SWI), and average total cost of samples and switches (TOT), if sampling and switching costs are equal. This table considers the DM configuration.

\begin{tabular}{lcccc}
\hline & OBS & PCS & SWI & TOT \\
\hline$H A K+$ & 2392 & 0.980 & 2107 & 4499 \\
$C M S$ & 3472 & 0.993 & 30 & 3502 \\
\hline
\end{tabular}

Table 2: Average number of required observations (OBS), observed PCS, average number of required switches (SWI), and average total cost of samples and switches (TOT), if sampling and switching costs are equal. This table considers the MIM configuration.

\begin{tabular}{lcccc}
\hline & OBS & PCS & SWI & TOT \\
\hline$H A K+$ & 1270 & 0.999 & 985 & 2255 \\
$C M S$ & 1833 & 1.000 & 29 & 1862 \\
\hline
\end{tabular}

Since $C M S$ was proven valid, the PCS performance in Tables 1 and 2 is expected to be better than the nominal 0.95. We observe this to be true in both cases. Moreover, $C M S$ commonly provides a higher PCS than $H A K+$, which is a result of the extra samples needed to limit switches during the procedure's comparison phase.

The last column of Tables 1 and 2 show why CMS is a competitive procedure when the cost of switches is counted. CMS requires 30 switches in DM and less, 29, in MIM. The other procedure, $H A K+$, can require thousands of switches, as every stage of sampling consists of as little as one observation from each system in contention.

\section{CONCLUSIONS}

We present the Constrained Minimal Switching, CMS, procedure that minimizes the number of switches between simulated systems while finding the best constrained system. This is desirable, as the cost of switching can be expensive. We prove the validity of this procedure and present experimental results that suggest that $C M S$ is an efficient option if the cost of switching is equal to the cost of sampling. The full proof of validity, additional experimental results, and a study into the implementation of common random numbers within the $C M S$ procedure can be found in Healey et al. (2010c).

\section{ACKNOWLEGMENTS}

This research was supported by the National Science Foundation under Grant Numbers CMMI-0400260 and CMMI0644837. The second author was also supported by the National Science Foundation under Grant Number CMMI-0856600.

\section{REFERENCES}

Andradóttir, S., and S.-H. Kim. 2010. Fully sequential procedures for comparing constrained systems via simulation. Naval Research Logistics, 57(5):403-421.

Batur, D., and S.-H. Kim. 2010. Procedures for feasibility check when the number of systems or constraints is large. To appear in ACM Transactions on Modeling and Computer Simulation.

Chen, H.C., C.H. Chen, and E. Yücesan. 2000. Computing efforts allocation for ordinal optimization and discrete event simulation. IEEE Transactions on Automatic Control, 45:960-964.

Chick, S.E. 2006. Subjective probability and Bayesian methodology. In Handbooks in Operations Research \& Management Science: Simulation, eds. S.G. Henderson and B.L. Nelson, 225-257. North-Holland: Amsterdam.

Chick, S.E., and K. Inoue. 2001. New two-stage and sequential procedures for selection the best simulated system. Operations Research, 49:732-743.

Healey, C.M., S. Andradóttir, and S.-H. Kim. 2010a. A dormancy framework for efficient comparison of constrained systems. Technical Report. H. Milton Stewart School of Industrial and Systems Engineering, Georgia Institute of Technology, Atlanta, GA 30332. 
Healey, C.M., S. Andradóttir, and S.-H. Kim. 2010b. Fully sequential selection procedures in simulations with multiple constraints. Technical Report. H. Milton Stewart School of Industrial and Systems Engineering, Georgia Institute of Technology, Atlanta, GA 30332.

Healey, C.M., S. Andradóttir, and S.-H. Kim. 2010c. A minimal switching procedure for constrained ranking and selection under independent or common random numbers. Technical Report. H. Milton Stewart School of Industrial and Systems Engineering, Georgia Institute of Technology, Atlanta, GA 30332.

Hong, L.J. and B.L. Nelson. 2005. The tradeoff between sampling and switching: New sequential procedures for indifference-zone selection. IIE Transactions 37:623-634.

Kabirian, A. and S. Ólafsson. 2009. Selection of the best with stochastic constraints. In Proceedings of the 2009 Winter Simulation Conference, eds. M.D. Rossetti, R.R. Hill, B. Johansson, A. Dunkin, and R.G. Ingalls, 584-589. Institute of Electrical and Electronics Engineers, Piscataway, New Jersey.

Kim, S.-H., and B.L. Nelson. 2001. A fully sequential procedure for indifference-zone selection in simulation. $A C M$ Transactions on Modeling and Computer Simulation, 11:251-273.

Kim, S.-H., and B.L. Nelson. 2006. Selecting the best system. In Handbooks in Operations Research \& Management Science: Simulation, eds. S.G. Henderson and B.L. Nelson, 501-534. North-Holland: Amsterdam.

Law, A., and D. Kelton. 2000. Simulation Modeling and Analysis, third edition. McGrawHill: New York.

Morrice, D.J. and J. Butler. 2006. Ranking and selection with multiple "targets". In Proceedings of the 2006 Winter Simulation Conference, eds. L.F. Perrone, F.P. Wieland, J. Liu, B.G. Lawson, D.M. Nicol, and R.M. Fujimoto, 222-230. Institute of Electrical and Electronics Engineers, Piscataway, New Jersey.

Osogami, T. 2009. Finding Probably Best Systems Quickly via Simulations. ACM Transactions on Modeling and Computer Simulation. 19(3):12:1-19.

Pujowidianto, N.A., L.H. Lee, C.-H. Chen, and C.M. Yap. 2009. Optimal computing budget allocation for constrained optimization. In Proceedings of the 2009 Winter Simulation Conference, eds. M.D. Rossetti, R.R. Hill, B. Johansson, A. Dunkin, and R.G. Ingalls, 574-583. Institute of Electrical and Electronics Engineers, Piscataway, New Jersey.

Rinott, Y. 1978. On two-stage selection procedures and related probability-inequalities. Communications in StatisticsTheory and Methods, A7:799-811.

Szechtman, R. and E. Yücesan. 2008. A new perspective on feasibility determination. In Proceedings of the 2008 Winter Simulation Conference, eds. S.J. Mason, R.R. Hill, L. Mönch, O. Rose, T. Jefferson, J.W. Fowler, 273-280. Institute of Electrical and Electronics Engineers, Piscataway, New Jersey.

\section{AUTHOR BIOGRAPHIES}

CHRISTOPHER M. HEALEY is an engineer at APC by Schneider Electric. He received his Ph.D. from the H. Milton Stewart School of Industrial and Systems Engineering at the Georgia Institute of Technology in 2010. His research interests lie in ranking and selection, simulation output analysis, and applications to data center energy and cooling efficiency. His email address is <chris.healey@apc.com>.

SIGRÚN ANDRADÓTTIR is a Professor in the H. Milton Stewart School of Industrial and Systems Engineering at the Georgia Institute of Technology. Prior to joining Georgia Tech, she was an Assistant Professor and later an Associate Professor in the Departments of Industrial Engineering, Mathematics, and Computer Sciences at the University of Wisconsin - Madison. She received her Ph.D. in Operations Research from Stanford University in 1990. Her research interests include simulation, applied probability, and stochastic optimization. She is a member of INFORMS and served as Editor of the Proceedings of the 1997 Winter Simulation Conference. She was the Simulation Area Editor of Operations Research Letters from 2002 to 2008, and has served as Associate Editor for various journals. Her e-mail address is<sa@gatech.edu>, and her web page is <http://www.isye.gatech.edu/faculty/sa>.

SEONG-HEE KIM is an Associate Professor in the H. Milton Stewart School of Industrial and Systems Engineering at the Georgia Institute of Technology. She received her Ph.D. in Industrial Engineering and Management Sciences from Northwestern University in 2001. Her research interests include ranking and selection, optimization via simulation, quality control, simulation output analysis, and applications of simulation methods to environmental management. She is a member of INFORMS, and serves as an associate editor in the simulation area of Operations Research and the OR/simulation area of The American Statistician. She also served on the editorial board of the Simulation Department of IIE Transactions. Her e-mail and web addresses are <skim@isye.gatech.edu> and <www. isye.gatech.edu/ skim/> respectively. 